\title{
Randomized, Clinical Trial on Diathermy and Scalpel Incisions in Elective General Surgery
}

\author{
Altaf Ahmed Talpur ${ }^{1, *}$; Abdul Basir Khaskheli ${ }^{1}$; Nandlal Kella ${ }^{1}$; Akmal Jamal ${ }^{1}$ \\ ${ }^{1}$ Department of Surgery, Liaquat University of Medical and Health Sciences, Jamshoro, Pakistan \\ ${ }^{*}$ Corresponding Author: Altaf Ahmed Talpur, Department of Surgery, Liaquat University of Medical and Health Sciences, Jamshoro, Pakistan. Tel: $+92-3002185479,+92-2134570819$, \\ E-mail:altafktalpur@yahoo.com \\ Received: August 7, 2013; Revised: November 4, 2013; Accepted: November 9, 2014
}

\begin{abstract}
Background: Since a long time skin incisions have routinely been made with scalpels. Now a day there is a shift in trend from this method to electrosurgical skin incisions. However, fear of bad scars and improper wound healing has prevented its wide spread use. This Study aimed to compare both methods of skin incisions for different variables.

Objectives: The aim of this study was to examine incisional time, blood loss during incision and postoperative wound complications and pain with both methods of skin incision.

Patients and Methods: A prospective, comparative and randomized study was conducted at different hospitals of Hyderabad and Nawabshah, Pakistan from $1^{\text {st }}$ of December 2009 to $30^{\text {th }}$ of November 2011. The study included patients of either sex above the age of five years with general surgical pathology who were to undergo surgery. these candidates were randomly put into two groups. In Group A patients incision was made with a scalpel and in group B with diathermy. Data was analyzed for age, sex, comorbid illness, incisional time, blood loss during incision making and postoperative pain and wound complications.

Results: A total of 283 patients completed the follow-up and were included in the final analysis. Group A comprised of $143(50.53 \%)$ patients; $83(58 \%)$ males and 60 ( $42 \%$ ) females with a mean age of 36.03 years. Amongst the 140 patients of group B, there were 85 (60.7\%) males and 55 (39.3\%) females with a mean age of 36.52 years. Twenty-five (17.48\%) patients of group A and 27 (19.28\%) of group B had comorbid illnesses. Mean incision time was $8.9025-\mathrm{sec} / \mathrm{cm}^{2}$ for group A and $7.3057 \mathrm{sec} / \mathrm{cm}^{2}$ for group B patients. Mean blood loss during incision making was $1.8262 \mathrm{~mL} / \mathrm{cm}^{2}$ and $1.1346 \mathrm{~mL} / \mathrm{cm}^{2}$ for group A and B patients, respectively. Pain was 5.2957 for group A patients on day one and 3.1181 for group B patients. Pain score was 2.1049 and 1.6206 on day two and 0.8191 and 0.7192 on day five, for group A and B patients, respectively. Postoperative wound complications were noticed in 26 (18.18\%) patients of group A and 22 (15.71\%) patients of group B.

Conclusions: Diathermy incision is a safe and expedient technique. It takes less time than scalpel incision and loss of blood is also lower during incision. Diathermy is associated with lesser post-operative pain and complications than the scalpel incision. Diathermy should be method of choice in general elective surgery.

Keywords:Diathermy; Scalpel; Complications; Pain, Postoperative
\end{abstract}

\section{Background}

Traditionally scalpels are used for making skin incisions that produce little damage to surrounding tissues (1). However, there has been a continuous surge in identifying other methods of skin incision and in the recent years electro surgical instruments have achieved great attention in this regard. There has been a widespread use of diathermy for homeostasis but fear of production of large scars and improper tissue healing has restricted their usage in making skin incisions $(2,3)$. Electrodes used in making diathermy incision generate a pure sinusoidal current, which produces cleavage in tissue planes without creating damage to the surrounding areas. This is one of the reasons of less damage inflicted to the tissues leading to minimal scar formation $(2,4)$.

At the same time, use of diathermy in skin incisions reduces bleeding and makes the incision quicker (5) but there are no differences in wound burst strength. Previously, it has been reported that there is a greater rate of infection with diathermy incisions than with scalpel incisions (6). Many studies in the past have evaluated perioperative blood loss, postoperative wound pain and wound healing in a selected group of patients, mainly with midline laparotomy incisions $(4,7)$. There have been limited studies comparing diathermy incisions with conventional scalpel incisions amongst all types of elective surgical procedures.

\section{Objectives}

This study focused on all general surgical operations and compared incisional time, blood loss during incision making, postoperative pain and wound complications for both methods of skin incision.

\section{Patients and Methods}

This was a randomized controlled trial conducted at different hospitals of Hyderabad and Nawabshah Pakistan from $1^{\text {st }}$ of December 2009 to $30^{\text {th }}$ of November 2011. It included all patients of either sex above the age of five years with general surgical pathology, who were to undergo surgery. Patients aged less than five years, with serious comor-

Copyright (C) 2015, Iranian Red Crescent Medical Journal. This is an open-access article distributed under the terms of the Creative Commons Attribution-NonCommercial 4.0 International License (http://creativecommons.org/licenses/by-nc/4.0/) which permits copy and redistribute the material just in noncommercial usages, provided the original work is properly cited. 
bid illnesses like severe cardiac (ASA-3) or airway disease or liver disease were excluded. Also patients who presented in acute surgical illnesses with gross infection found at operative site were excluded. Patients who did not attend follow-up sessions were also excluded from the final analysis.

Random distribution of patients was done utilizing the blocked method. These patients were divided into blocks of two with scalpel group given block A and diathermy group block $B$. The first patient was allocated to block $A$ and the second patient to block B. These patients were admitted to the hospitals and a detailed history was obtained from them especially data related to age, sex, comorbid illness, and any previous surgery. Thorough examination was conducted to determine variables such as general health, weight, anemia, jaundice and any previous scars. Investigations for general fitness, including variables such as blood Complete Picture (Blood CP), blood sugar and blood urea, were performed. When required electrocardiogram (ECG) and chest $\mathrm{x}$-ray were also performed for the same purpose. In addition, investigations like ultrasound, CT-scan, intravenous urography, gastroduodenoscopy, contrast studies of gut, thyroid scan, mammography and fine needle aspiration cytology (FNAC) were performed to establish diagnosis. Patients were briefed about the diagnosis and procedure to be performed. Those who met the eligibility criteria were invited to participate in the study and were informed about both methods of skin incisions. Advantages and disadvantages of both types of incisions were explained and permission was granted from the patients. Those who refused were not enrolled in the study and those who consented to participate were enrolled and assured that their participation is voluntary with no harms to them in terms of getting due treatment. They were also given the right to withdraw from the study without any reason.

Standard prophylactic antibiotics were given to all patients according to the site of injury and patient's age at the time of induction of anesthesia, and were continued for one to three days depending on the type of surgery. Skin and subcutaneous tissue was incised with a surgical blade for group A patients and with diathermy for group $B$ patients. Hemostasis was secured with the coagulation mode of diathermy or with ligatures. An electrosurgical instrument brand Valley lab force 40 was used for the entire study. It was set at $417 \mathrm{KHz}$ sinusoidal current. The instrument was checked and calibrated periodically according to service manual guidelines, by the bio-medical engineering department.

There was more than one observer during the whole study period. Different interns were trained and allocated to observe different components of the process and record the data for each individual patient. Each intern recorded data on one variable and only one observer was allocated to single variable. Subcutaneous tissue was closed with chromic 2/0 interrupted stitches and skin stitches were applied in a subcuticular pattern with Prolene 2/0. Postoperatively both groups of patients received intramuscular diclofenac sodium three times a day supplemented by intravascular infusion of tramadol as necessary. As patients tolerated oral feeding well they were shifted to oral diclofenac sodium three times daily. In none of the patients subcutaneous drains were placed.

As patients tolerated oral feeding and became mobile they were discharged from the hospital. The wound was checked on the fifth postoperative day if the patient had remained in the hospital. It was checked earlier when the dressing of the patient became soaked or the patient developed fever or tachycardia with no other source of fever or tachycardia noticed. Patients were advised to attend outpatient clinics for removal of stitches on the $10^{\text {th }}$ postoperative day. Follow-up visits were advised at one, three and six months.

Sample size was calculated using the G-Power software version 3.0.10; with an effect size of 0.4 and $95 \%$ power at $5 \%$ level of significance, a total sample of 272 patients were required with 136 participants in each group. This was inflated to 300 to account for those that discontinued follow-ups. All this data was recorded on a pro forma. Data was calculated and analyzed for categorical and continuous variables such as age, sex, comorbid illness, incisional time, blood loss during incision making and postoperative pain and wound complications for both methods of skin incision. Incision time, blood loss during incision making and postoperative wound pain were analyzed with Student's t-test and wound complications were compared for the two groups using the chi-square test. P values of less than 0.05 were considered significant. All analysis was done using the SPSS software, version 12.0.

The tested hypothesis was that skin incision with a conventional surgical blade has better results in terms of the above-mentioned variables than diathermy incision. Incision time was calculated from the start of skin incision until deep fascia, aponeurosis or lump reached with complete hemostasis. Incision length and depth was measured using sterilized calibrated scales. Blood loss during incision was measured weighing the swabs pre and postoperatively. Wound pain was calculated by the verbal rating score(VRS). Wound healing was classified using the Southampton wound grading system; GO: normal wound healing, G1: normal healing with mild bruising or erythema, G2: erythema plus other signs of inflammation, G3: clear or serosanguinous discharge, and G4: Purulent discharge.

\section{Results}

Initially 300 patients with different pathologies related to general surgery were included in the study. However, seven patients from group $A$ and ten patients from group $B$ did not complete the follow up and were excluded from the study. A total of 283 patients of different pathologies related to general surgery who completed the follow-up period were finally included in the final analysis. Group A comprised of 143 (50.53\%) patients whose incision was made with a scalpel; including 83 (58\%) males and 60 (42\%) females. Mean age of this group of patients was 36.03 years, SD of \pm 13.24 years. Amongst the 140 patients 
TalpurAA et al.

of group B whose incision was made with diathermy, there were 85 (60.7\%) males and 55 (39.3\%) females. Mean age of group B patients was 36.52 years, $S D \pm 12.50$ years. The total numbers of male and female patients were 168 and 115, respectively, making a 1.46:1.00 ratio. The most common operations performed in this study was on the kidneys and upper ureters, performed on 76 (26.85\%) patients, followed by 61 (21.55\%) patients of hernias (Table 1 ). Comorbid illnesses were noticed in 25 (17.48\%) and 27 (19.28\%) patients of group A and B, respectively. Their distribution was as follows; Group A: diabetes mellitus (DM): 16 (11.21\%), hypertension (HTN): one (0.7\%), both DM and HTN: three (2.1\%), chronic obstructive pulmonary disease (COPD): four (2.8\%), chronic live disease (CLD): one (0.7\%); Group B: DM:17(12.1\%), HTN 2 (1.4\%), Both DM and HTN: 5 (3.6\%), COPD: 3 (2.1\%). We compared the two groups for baseline characters such as age, sex and presence of comorbid conditions and the groups were similar with respect to these variables as no statistically significant difference was found.

Mean incision time was $8.9025 \mathrm{sec} / \mathrm{cm}^{2}(\mathrm{SD} \pm 1.3666 \mathrm{sec} /$ $\left.\mathrm{cm}^{2}\right)$ for group A and $7.3057 \mathrm{sec} / \mathrm{cm}^{2}\left(\mathrm{SD} \pm 0.9677 \mathrm{sec} / \mathrm{cm}^{2}\right)$ for group B patients. Similarly mean incision blood loss was also found to be significantly higher in group A i.e. $1.8262 \mathrm{~mL} / \mathrm{cm}^{2}$ (SD $\pm 0.2984 \mathrm{~mL} / \mathrm{cm}^{2}$ ) compared to 1.1346 $\mathrm{mL} / \mathrm{cm}^{2}\left(\mathrm{SD} \pm 0.3399 \mathrm{~mL} / \mathrm{cm}^{2}\right)$ in group B patients. Postoperative pain was assessed by VRS on day one, two, and five. It was significantly higher in group A. Mean hospital stay was 62.8940 ( $S D \pm 45.007$ ) hours in group A and 56.6430 (SD \pm 42.1363) hours among group $B$ patients. This difference was also statistically significant (Table 2). Among wound complications, 26 (18.18\%) patients from group A and 22 (15.71\%) patients from group B developed wound complications. Erythema of wound margin (G: 1) was found in eight (5.6\%) patients of group A and four (2.9\%) patients of group B. Overall no statistically significant differences were seen regarding wound complications for the two groups (Table 3).

\begin{tabular}{lccc}
\hline Table 1. Pathological Viscera Found in the Study $(\mathrm{n}=283)^{\mathrm{a}}$ & & & \\
\hline Pathological Viscera & Scalpel Incision & Diathermy Incision & Total \\
\hline Kidneys and upper ureters & $37(25.9)$ & $39(27.9)$ & $76(26.85)$ \\
Hernias & $29(20.3)$ & $32(22.9)$ & $61(21.55)$ \\
Gall bladder and biliary tree & $21(14.7)$ & $23(16.4)$ & $44(15.54)$ \\
Lower ureters, urinary bladder and prostate & $14(9.8)$ & $12(8.6)$ & $26(9.18)$ \\
Surface swelling & $12(8.4)$ & $10(7.1)$ & $22(7.77)$ \\
Gastrointestinal tract & $10(7.0)$ & $7(5.0)$ & $17(6.007)$ \\
Scrotal pathology & $6(4.2)$ & $5(3.6)$ & $11(3.88)$ \\
Thyroid pathology & $5(3.5)$ & $3(2.1)$ & $8(2.82)$ \\
Breast pathology & $4(2.8)$ & $3(2.1)$ & $7(2.47)$ \\
Uterine and ovarian pathology & $3(2.1)$ & $4(2.9)$ & $7(2.47)$ \\
Retro peritoneal mass & $2(1.4)$ & $2(1.4)$ & $4(1.41)$ \\
Total & $143(50.53)$ & $140(49.46)$ & $283(100)$ \\
\hline
\end{tabular}

${ }^{\mathrm{a}}$ Data are presented as No.(\%).

\begin{tabular}{|c|c|c|c|c|}
\hline Parameter & Value $^{\mathrm{a}}$ & Minimum & Maximum & PValue \\
\hline Incisional Time, sec/cm² & & & & $<0.001$ \\
\hline Scalpel & $8.9025 \pm 1.3666$ & 5.32 & 11.98 & \\
\hline Diathermy & $7.3057 \pm 0.9677$ & 4.36 & 10.87 & \\
\hline Incisional Blood loss, $\mathrm{mL} / \mathrm{cm}^{2}$ & & & & $<0.001$ \\
\hline Scalpel & $1.8262 \pm 0.2984$ & 1.23 & 2.45 & \\
\hline Diathermy & $1.1346 \pm 0.3399$ & 0.62 & 1.86 & \\
\hline Pain, day 1 & & & & $<0.001$ \\
\hline Scalpel & $5.2957 \pm 0.8350$ & 3.89 & 6.89 & \\
\hline Diathermy & $3.1182 \pm 0.1719$ & 2.82 & 3.87 & \\
\hline Pain, day 2 & & & & $<0.001$ \\
\hline Scalpel & $2.1049 \pm 0.1163$ & 1.87 & 2.48 & \\
\hline Diathermy & $1.6206 \pm 0.1073$ & 1.40 & 1.90 & \\
\hline Pain, day 5 & & & & $<0.001$ \\
\hline Scalpel & $0.8191 \pm 0.09469$ & 0.55 & 1.02 & \\
\hline Diathermy & $0.7129 \pm 0.09604$ & 0.53 & 0.91 & \\
\hline Mean Hospital Stay ${ }^{b}, h$ & & & & 0.229 \\
\hline Scalpel & $62.8940 \pm 45.0097$ & 0.19 & 180.50 & \\
\hline Diathermy & $56.6430 \pm 42.1363$ & 0.20 & 178.45 & \\
\hline
\end{tabular}

\footnotetext{
a Data are presented as Mean \pm SD.
}

b Mann Whitney U test was used as this variable did not have a normal distribution. 
TalpurAA et al.

\begin{tabular}{lcccc}
\hline \multicolumn{4}{l}{ Table 3. Comparative Analysis of Postoperative Wound Complications in Diathermy and Scalpel Incision Groups $(\mathrm{n}=283)$} \\
\hline Wound Complication & Scalpel Incision $^{\mathrm{a}}$ & Diathermy Incision $^{\mathrm{a}}$ & Total & P Value \\
\hline Nil & $117(81.8)$ & $118(84.3)$ & $235(83.03)$ & $0.098^{\mathrm{b}}$ \\
G1 & $8(5.6)$ & $4(2.9)$ & $12(4.24)$ & $0.056^{\mathrm{C}}$ \\
G2 & $9(6.3)$ & $2(1.4)$ & $11(3.88)$ & \\
G3 & $5(3.5)$ & $10(7.1)$ & $15(5.30)$ & \\
G4 & $4(2.8)$ & $6(4.3)$ & $10(3.53)$ & \\
\hline
\end{tabular}

a Data are presented as No. (\%).

b P-value when all the categories of wound complications compared.

c P-value when "Nil" category was excluded.

\section{Discussion}

In an era of explosive anesthetic agents, electro surgical instruments were used only selectively in human surgery. After the introduction of halothane as an anesthetic agent, diathermy became increasingly used to control bleeding and for dissection of tissue planes. However, it is still infrequently used for making skin incisions. The reluctance in the use of skin incision is due to the fear that electro surgical instruments create increased amounts of necrotic tissue within the wound which may increase the chances of wound infection leading to delayed wound healing and excessive scarring (2, 8-10).

After the introduction of oscillator units, which produce pure sinusoidal current, there has been an increasing trend in the use of diathermy for making skin incisions. In the recent years, many studies have been conducted on both methods of skin incision, which showed less operating time, diminished loss of blood, and reduced early pain and fewer requirements of analgesic drugs after surgery using the diathermy method of skin incision when compared to scalpel incision (2). In one experimental study, conducted on rats, it was shown that wound incisions made with a cold scalpel had more rapid tensile strengths as compared to diathermy or harmonic scalpels (8).

Ly et al. (11) in their systemic review and meta-analysis of fourteen randomized trials comprising of 2541 patients (1267 undergoing skin incision by cutting diathermy and 1274 by scalpel), found that diathermy may offer significant advantages in many variables including, operative blood loss, incision time and postoperative pain. They noticed significantly reduced amounts of blood loss (mean difference of $0.72 \mathrm{~mL} / \mathrm{cm}(2) ; \mathrm{P}<0.001)$ and shorter incision times (mean difference of 36 seconds; $\mathrm{P}<0.001$ ) with diathermy incisions as compared to scalpel incisions.

In our study, diathermy mode of skin incision took less time and led to less loss of blood i.e. $7.3057 \mathrm{sec} / \mathrm{cm}^{2}$ and $1.1346 \mathrm{~mL} / \mathrm{cm}^{2}$, respectively compared to scalpel incisions in which measurement of these variables were $8.9025 \mathrm{sec} / \mathrm{cm}^{2}$ and $1.8262 \mathrm{~mL} / \mathrm{cm}^{2}$, respectively. These findings are consistent with findings reported by Ly et al. and another local study. In a study by Shamim M (3), incision time was $6.2453 \mathrm{sec} / \mathrm{cm}^{2}$ and incisional blood loss was $1.75 \mathrm{~mL} / \mathrm{cm}^{2}$ in the scalpel group, which was shorter than our study.

In the study by Aird et al. (12) from Canada, they performed a systematic electronic literature search using two electronic databases (MEDLINE and PubMed), and the methodological quality of included publications was evaluated. Six randomized control trials (RCTs), which compared the electrocautery method of skin incision ( $n$ $=606)$ with scalpel incision $(n=628)$, were analyzed. They noticed less incisional blood loss and reduced operating time with the electrocautery method of skin incision. Gilmore and colleagues (13) at Dublin, Ireland, compared diathermy and scalpel incisions for hemiarthroplasty. In their study certain variables such as age, sex and preoperative aspirin use were similar in both groups. Scalpel incisions produced per operative blood loss, which was over $30 \%$ of the total operative blood loss as compared to $18.5 \%$ in incisions made with diathermy.

Chalya et al. (14) in their study on diathermy versus scalpel incisions in elective midline laparotomy at Tanzania showed the mean incision time with scalpel was 9.21 \pm $1.40 \mathrm{sec} / \mathrm{cm}^{2}$ in comparison to $7.84 \pm 0.82 \mathrm{sec} / \mathrm{cm}^{2}$ with diathermy incisions. The difference between the two groups with respect to the mean incision time was statistically significant $(\mathrm{P}=0.001)$. The mean loss of blood was $1.62 \pm 0.14 \mathrm{~mL} / \mathrm{cm}^{2}$ for scalpel incisions and $1.12 \pm 0.20 \mathrm{~mL} /$ $\mathrm{cm}^{2}$ for diathermy incisions thus significantly less bleeding was noticed with diathermy incisions $(\mathrm{P}=0.012)$.

Elective midline laparotomy incisions made with diathermy have significant benefits over scalpel incisions in terms of decreased incision time and reduced blood loss as shown in many studies performed by Siraj et al. (10), Gilmore et al. (13) and Shivagouda et al. (15). In his study Sheikh (16) noticed significantly shorter incision times and reduced blood loss in diathermy skin incisions. In one study by Kearns and colleagues (2) it was found that diathermy produces significantly less postoperative pain on the first and second postoperative day when compared to scalpel incisions. From the third postoperative day onwards, severity of pain after surgery became significantly different between the two groups. In this study postoperative pain on the first and second postopera- 
tive days was higher in the scalpel group (i.e. 5.2957 and 2.1049) when compared to the diathermy group (3.1181 and 1.6206). There was no significant difference in pain of both groups on subsequent days.

Chrysos et al. (17) in their prospective study comparing diathermy and scalpel incisions in tension free inguinal hernioplasty noted lower VRS with diathermy incisions during the initial two postoperative days. They found that immediate tissue and nerve necrosis with diathermy might be due to cell vaporization, which doesn't significantly affect the nearby structures. Chalya et al. (14) revealed significantly reduced mean VAS with diathermy incisions as compared to scalpel incisions on postoperative day one $(\mathrm{P}=0.001)$, two $(\mathrm{P}=0.011)$ and three $(\mathrm{P}=$ 0.021). Intramuscular analgesic requirements were also significantly lower with diathermy incisions than scalpel incisions $(P=0.021)$. The difference between the two groups was not statistically significant $(P=0.243)$.

In their study Aird et al. (12) noted that electrocautery significantly reduced postoperative wound pain. Results of our study are consistent with other studies by Siraj et al. (10), Gilmore et al. (13) and Shivagouda et al. (15), which showed that elective midline laparotomy incisions made with diathermy had significant benefits compared to scalpel incisions in terms of reduced early postoperative pain and analgesic requirements.

In our study, postoperative wound complications were slightly higher in patients of the scalpel group (18.18\%) than diathermy group (15.71\%). Similar results were reported by Kearns et al. (2), Shamim (3), and Chrysos et al. (17). In the study of Franchi et al. (18) on gynecological oncological patients demonstrated that scalpel incisions produced severe wound complications in a greater number of patients as compared to incisions made by electrocautery (8.531 vs. 1.433, P = 0.05). However, after adjustment of confounding variables like age and body mass index there was no difference in wound complications between the two groups.

Eren et al. (19) from Istanbul compared wound complications associated with scalpel and electrocautery in patients operated for gastrointestinal malignancies with different incision methods. Their study revealed no significant statistical difference in wound infection or incisional hernia between the two groups $(\mathrm{P}>0.05)$. Therefore, in their study the choice of incision method depended upon the surgeon's preference. In their study Aird and colleagues (12) noticed no significant difference in wound infection or scar formation between the two methods of skin incision. None of the patient in both groups developed wound infection or dehiscence as reported by Gilmore and their colleagues (13). However wound oozing was noticed in the scalpel group in four patients that were treated conservatively. Post-operative complications were not significantly different amongst the two groups as reported by the study of Chalya et al. (14).

Groot and Chappell (20) noticed wound infections in $15 \%$ (38/250) of patients when incision was made by a scal- pel and in 12\% (30/342) of patients with incisions made by cautery. They noticed no difference in wound infections amongst patients of different age and genders, level of obesity, those using steroids and cases with diabetes. Also duration of preoperative stay at the hospital, duration of surgery, prophylactic usage of antibiotics and presence of drains produced no significant difference in wound infection of both groups. Chalya et al. (14) reported that the mean length of hospital stay (LOS) in both groups was $14.63 \pm 6.36$ (scalpel group $12.34 \pm 34$ and diathermy group $11.78 \pm 6.48$ days). The mean LOS did not differ significantly between the two groups $(\mathrm{P}=0.834)$. This data shows that electrocautery has significant advantages in creating incisions in surgery patients. The surgeon must be properly trained and thoroughly familiar with the effects of local wound environment on healing (21).

Further, increased prevalence of blood borne diseases like hepatitis $\mathrm{C}$ and HIV infection favor the use of electrocautery in skin incisions thereby keeps the scalpel away from the operative field (2). Our study is one of the few attempts towards comparing the effectiveness of the two incision techniques. We included participants from two different cities and included both public and private health facilities, which provided us with a diverse group of participants. We assigned observers for specific measurements, which add to the accuracy of the results as assigning multiple measurements at the time of procedure to one individual could influence their observations.

However as with any study there are certain limitations in our study as well. First we could not assign more than one person to assess the clinical outcomes due to budgetary limitations but strict adherence to the protocol was ensured to achieve reliable and consistent measurements. Secondly, private sector hospitals were selected conveniently as permission from most private hospitals was not granted; however, the included hospitals were large private sector hospitals with large catchment areas. Finally, 17 (5.6\%) of the participants were lost to follow up, but when compared they were not different from those who completed the follow-up (data not shown).

Electrosurgical incision is safe for both patients as well as for surgeon. It requires less time, there is less blood loss during skin incisions, and produces decreased postoperative pain and wound complications. At the same time there is a significant number of Hepatitis B and C patients who require surgery. The use of electrocautery in skin incision keeps scalpels away from the operative field thereby decreasing chances of transmission of these and other lethal diseases to the operating team. We recommend further studies in this regard to confirm the reliability of this method of skin incision and if found fruitful, it may be adopted as a hospital policy for making skin incisions.

\section{Acknowledgements}

We greatly appreciate and acknowledge all patients who participated in this study. 


\section{Authors' Contributions}

Dr Altaf Ahmed Talpur: conception, design and acquisition of data. Dr Abdul Basir Khaskheli: analysis and interpretation of data. Dr Nandlal kella: drafting and critical revision of the manuscript. Prof. Akmal Jamal: final approval of the manuscript.

\section{Funding/Support}

All the expenses of this study were provided by the authors.

\section{References}

1. Johnson CD, Serpell JW. Wound infection after abdominal incision with scalpel or diathermy. BrJ Surg. 1990;77(6):626-7.

2. Kearns SR, Connolly EM, McNally S, McNamara DA, Deasy J. Randomized clinical trial of diathermy versus scalpel incision in elective midline laparotomy. BrJ Surg. 2001;88(1):41-4.

3. Shamim M. Diathermy vs. scalpel skin incisions in general surgery: double-blind, randomized, clinical trial. World J Surg. 2009;33(8):1594-9.

4. Dixon AR, Watkin DF. Electrosurgical skin incision versus conventional scalpel: a prospective trial. $J$ R Coll Surg Edinb. 1990;35(5):299-301.

5. Sebben JE. Electrosurgery principles: cutting current and cutaneous surgery--Part I.J Dermatol Surg Oncol.1988;14(1):29-31.

6. Madden JE, Edlich RF, Custer JR, Panek PH, Thul J, Wangensteen $\mathrm{OH}$. Studies in the management of the contaminated wound. IV. Resistance to infection of surgical wounds made by knife, electrosurgery, and laser. Am J Surg. 1970;119(3):222-4.

7. Hussain SA, Hussain S. Incisions with knife or diathermy and postoperative pain. BrJ Surg. 1988;75(12):1179-80.

8. Ozgun H, Tuncyurek P, Boylu S, Erpek H, Yenisey C, Kose H, et al. The right method for midline laparotomy: what is the best choice for wound healing? Acta Chir Belg. 2007;107(6):682-6.

9. Kumar V, Tewari M, Shukla HS. A comparative study of scalpel and surgical diathermy incision in elective operations of head and neck cancer. Indian J Cancer. 2011;48(2):216-9.

10. Siraj A, Gilani AAS, Dar MF, Raziq S. Elective midline laparotomy: Comparison of Diathermy and scalpel incisions. Professional Med J. 2011;18(1):106-11.

11. Ly J, Mittal A, Windsor J. Systematic review and meta-analysis of cutting diathermy versus scalpel for skin incision. Br J Surg. 2012;99(5):613-20.

12. Aird LN, Brown CJ. Systematic review and meta-analysis of electrocautery versus scalpel for surgical skin incisions. Am J Surg. 2012;204(2):216-21.

13. Gilmore M, McCabe JP, Kaar K, Curtin W. Diathermy versus scalpel incisions for hemiarthroplasty: A randomized prospective trial. Bone Joint Surg Br. 2004;86(SUPP II):129.

14. Chalya PL, Mchembe MD, Mabula JB, Gilyoma JM. Diathermy versus Scalpel incision in elective midline laparotomy: A prospective randomized controlled clinical study. East and Central Afric J Surg. 2013;18(1):71-7.

15. Shivagouda P, Gogeri BV, Godhi AS, Metgud SC. Comparing the efficacy of diathermy incision versus scalpel incision over skin in patients undergoing inguinal hernia repair: Prospective randomized control trial. Recent Research Sci Tech. 2010;2(8):44-7.

16. Sheikh B. Safety and efficacy of electrocautery scalpel utilization for skin opening in neurosurgery. BrJ Neurosurg. 2004;18(3):268-72.

17. Chrysos E, Athanasakis E, Antonakakis S, Xynos E, Zoras O. A prospective study comparing diathermy and scalpel incisions in tension-free inguinal hernioplasty. Am Surg. 2005;71(4):326-9.

18. Franchi M, Ghezzi F, Benedetti-Panici PL, Melpignano M, Fallo L, Tateo S, et al. A multicentre collaborative study on the use of cold scalpel and electrocautery for midline abdominal incision. Am J Surg. 2001;181(2):128-32.

19. Eren T, Balik E, Ziyade S, Yamaner S, Akyuz A, Bugra D. Do different abdominal incision techniques play a role in wound complications in patients operated on for gastrointestinal malignancies? "Scalpel vs. electrocautery". Acta Chir Belg. 2010;110(4):451-6.

20. Groot G, Chappell EW. Electrocautery used to create incisions does not increase wound infection rates. Am J Surg. 1994;167(6):601-3.

21. Peterson A. The use of electrosurgery in reconstructive and cosmetic maxillofacial surgery. Dent Clin North Am.1982;26(4):799-823. 